\title{
Cell-mediated Immunity in Patients Positive for Hepatitis-associated Antigen
}

\section{Summary}

Cell-mediated immunity to antigens prepared from both serum and liver of patients positive for hepatitis-associated antigen (H.A.A.) was measured by using the leucocyte migration test. Altogether, 43 patients with H.A.A.positive acute and chronic liver disease, eight with serum antibody to H.A.A., and 13 controls were studied. The cell-mediated immunity detected was specific for H.A.A. or other antigenic determinants of the associated infective agent and could be found only in patients with evidence of previous contact with H.A.A.

Cell-mediated immunity to the H.A.A.-positive test antigens was found in all but one of the patients with acute hepatitis, in about half of the patients with chronic aggressive hepatitis or cirrhosis, rarely in those with chronic persistent hepatitis, and in none of the apparently healthy carriers.

Our results support the hypothesis that the cellular immune response plays an important part in the clearance of the infective agent from H.A.A.-positive patients and in the pathogenesis of the associated liver cell injury.

\section{Introduction}

Hepatitis-associated antigen (H.A.A.) is a marker for the infective agent responsible for long incubation (type B) acute infective hepatitis (Giles et al., 1969; Krugman and Giles, 1970). Dudley et al. (1972) suggested that the liver cell damage in H.A.A.-positive patients may be related to the thymusdependent $(T)$ lymphocyte cell-mediated immune response against that infective agent. Which clinical course occurs in any H.A.A.-positive patient may then depend on variations in $T$ lymphocyte function. In the presence of normal $T$ lymphocyte function an acute hepatitis will be produced which will resolve completely with clearance of the infective agent. When specific $T$ lymphocyte function against the infective agent is absent both the infective agent and H.A.A. will persist with no evidence of liver cell damage. If $T$ lymphocyte function is impaired an intermediate course will result with incomplete removal of the infective agent and continuing liver cell damage.

After contact with a specific antigen the development of cellmediated immunity to that antigen is dependent on the production of sensitized $T$ lymphocytes which on subsequent exposure to the antigen are capable of recognizing and reacting with it. This lymphocyte-antigen interaction results in the lymphocyte producing a number of soluble factors which result in both the inactivation of antigen and in tissue damage. One of these soluble factors is macrophage migration inhibition factor. Thus the ability of $T$ lymphocytes to produce macrophage migration inhibition factor when incubated with an antigen can be used as an in-vitro index of cell-mediated immunity to that antigen. One such method of detecting the production of macrophage migration inhibition factor by sensitized $T$ lympho-

Department of Medicine, Royal Free Hospital, London WC1X 8LF F. J. DUDLEY, M.R.A.C.P., Honorary Assistant Lecturer

V. GIUSTINO, M.D., Research Fellow

S. SHERIOCK, M.D., F.R.C.P., Professor of Medicine cytes in the presence of specific antigens is the leucocyte migration inhibition test of Söborg and Bondixen (1967). We have used this test system to show cell-mediated immunity to antigens prepared from both serum and liver in 43 H.A.A.-positive and 21 H.A.A.-negative patients.

\section{Patients and Methods}

Altogether, 43 patients with H.A.A.-positive liver disease have been studied. They include eight with acute hepatitis, 21 with chronic aggressive hepatitis or cirrhosis, six with chronic persistent hepatitis, and eight carriers with no evidence of liver disease. Diagnosis was confirmed histologically in all except two of the carriers who both had normal liver function tests and persistent serum H.A.A. for at least six months.

Twenty-one healthy H.A.A.-negative subjects with and without antibody to H.A.A. were also studied.

Detection of H.A.A.-H.A.A. was found in serum specimens from all H.A.A.-positive patients at the time of study by counter immunoelectrophoresis (Gocke and Howe, 1970) using standard antibody (Fox et al., 1969).

Leucocyte Migration. - The method used was as described by Söborg (1971) with minor modifications. A $30-\mathrm{ml}$ sample of venous blood was drawn into plastic disposable syringes containing 600 units of heparin. After mixing with $5 \mathrm{ml}$ of $1 \%$ methyl cellulose the blood was allowed to sediment at $37^{\circ} \mathrm{C}$ for 30 minutes. The leucocyte-rich plasma was discharged and the contained cells washed three times in tissue culture medium (TC 199) and resuspended in about $0.5 \mathrm{ml}$ of the same medium. This suspension was aspirated into $1.4-\mathrm{mm}$ capillary tubes which were then sealed at one end by heating and centrifuged at 3,000 r.p.m. for three minutes. The capillary tubes were then cut 0.5 to $1 \mathrm{~mm}$ below the cell fluid interface and the part containing cells were fixed to the bottom of a $0.5-\mathrm{ml}$ culture chamber containing culture medium by silicone wax. The culture medium used consisted of a mixture of human sera and TC 199 in the ratio of 1 to 4. After addition of antigen the chambers were sealed excluding air. Sterile procedure was followed at all times. After incubation at $37^{\circ} \mathrm{C}$ for 18 hours the sera of leucocyte migration was measured by projection microscopy and paper planimetry. All cultures were performed in triplicate and only accepted if all were within $10 \%$ of each other. The average migration areas were calculated and the results expressed as the migration index by dividing the mean area of leucocyte migration in the test chamber containing antigen by the mean area of migration in the control chamber. A migration index between 0.8 and 1.2 represents no detectable cell-mediated immunity to the test antigen. Values less than 0.8 represent inhibition of migration and indicate the presence of cell-mediated immunity to the test antigen. The significance of values greater than 1.2 is not at present clear.

\section{PREPARATION OF ANTIGENS}

H.A.A.-positive Sera.-On the day of study both autologous and homologous H.A.A.-positive sera were collected under sterile conditions and used as antigen in the test culture chambers in place of the normal human serum in the control culture medium. Two-ml aliquots of sera were also stored at $-20^{\circ} \mathrm{C}$ for follow-up studies. 
H.A.A.-positive Liver.-Liver tissue obtained by percutaneous liver biopsy from H.A.A.-positive patients was homogenized after washing three times in culture medium. Concentration of the resultant suspension was adjusted so that $0.1 \mathrm{ml}$ of homogenate contained $50 \mu \mathrm{g}$ of protein. Aliquots of $0.6 \mathrm{ml}$ were stored at $-20^{\circ} \mathrm{C}$ and usually used within a week of preparation. When used as test antigen $0.05 \mathrm{ml}$ of homogenate was added to the culture medium producing a final concentration of $25 \mu \mathrm{g} / \mathrm{ml}$.

H.A.A.-negative Liver.-Histologically normal liver tissue obtained from H.A.A.-negative patients by either percutaneous or operative liver biopsy was prepared and used as that from H.A.A.-positive patients.

\section{LEUCOCYTE MIGRATION OF H.A.A.-POSITIVE PATIENTS}

With H.A.A.-positive Liver as Antigen.-Inhibition of leucocyte migration indicating the presence of cell-mediated immunity against this antigen was found in seven out of eight patients with acute hepatitis (Fig. 1). The patient in whom leucocyte migration was unaffected had a prolonged mild anicteric hepatitis. Cell-mediated immunity to this antigen could be found in only eight of 20 patients with chronic aggressive hepatitis or cirrhosis and in none of the 14 patients with chronic persistent hepatitis or apparently normal livers. Stimulation of leucocyte migration was seen in three of the patients with chronic liver disease. In 28 patients leucocytes were cultured in the presence

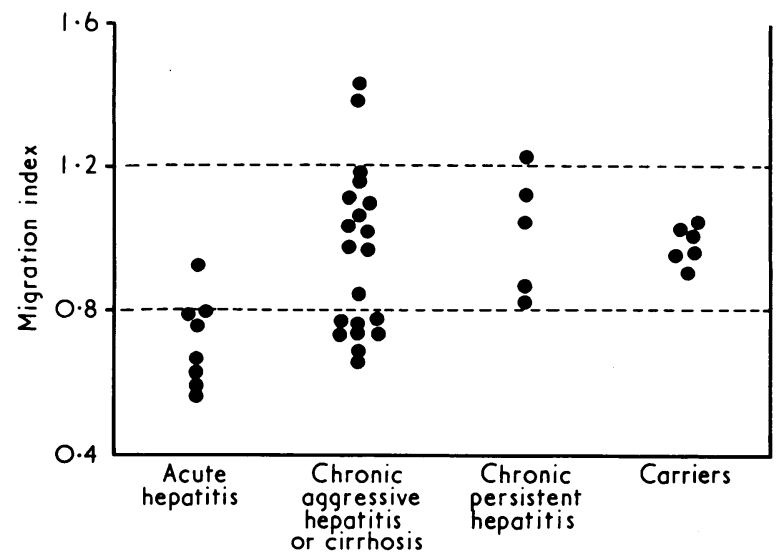

FIG. 1-Migration index of leucocytes from different groups of H.A.A.positive patients in presence of H.A.A.-positive liver antigen. Normal range (indicating no detectable cell-mediated immunity to the test antigen) represented by values between 0.8 and $1 \cdot 2$. Inhibition (indicating presence of cell-mediated immunity to test antigen) represented by values less than $0 \cdot 8$.

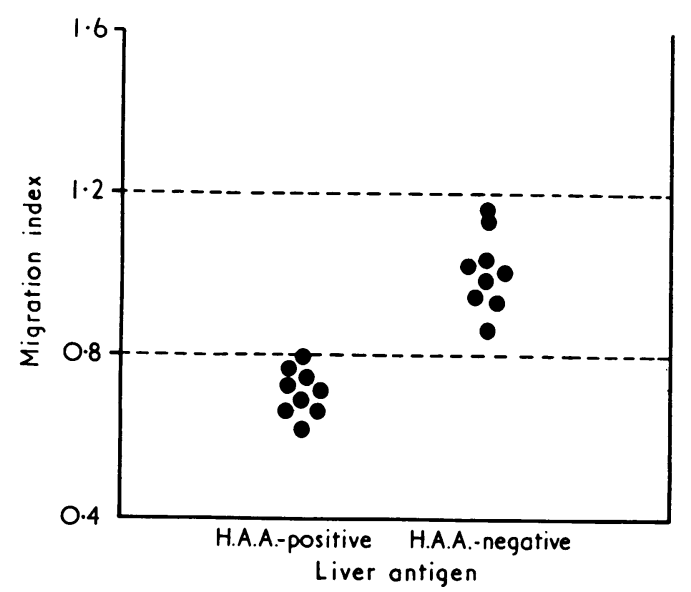

FIG. 2-Migration index of leucocytes from H.A.A.-positive patients in presence of either H.A.A.-positive or negative liver antigen. Normal range between broken rules. of both autc.ogous and homologous H.A.A.-positive liver antigen. Similar results were obtained with both antigens in 27 patients.

With H.A.A.-negative Liver as Antigen.-The leucocytes from nine patients who showed cell-mediated immunity to H.A.A.-positive liver had also been cultured on the same day in the presence of liver antigen prepared from H.A.A.-negative patients. None of these nine patients showed cell-mediated immunity to the H.A.A.-negative liver antigen (Fig. 2).

With H.A.A.-positive Sera as Antigen.-When autologous H.A.A.-positive serum was used as test antigen the results were similar to those obtained with H.A.A.-positive liver as antigen (Fig. 3). Inhibition of leucocyte migration indicating cell-

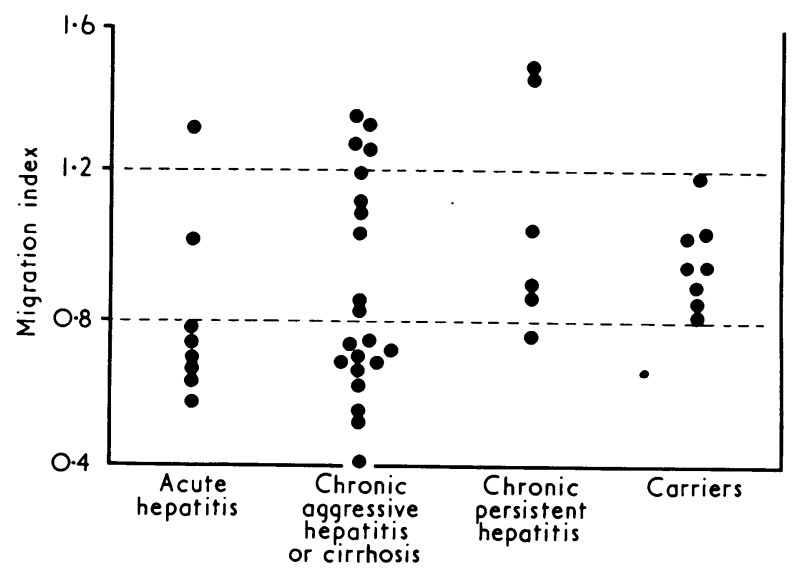

FIG. 3-Migration index of leucocytes from different groups of H.A.A.positive patients in presence of autologous H.A.A.-positive serum as antigen. Normal range between broken rules.

mediated immunity against the test antigens was found in six of eight patients with acute hepatitis, 11 of 21 with chronic aggressive hepatitis or cirrhosis, one of six with chronic persistent hepatitis, and in none of eight healthy carriers. Leucocyte migration was also measured in 20 of these patients in the presence of homologous H.A.A.-positive serum. The results were similar to those obtained with autologous sera in 18.

Changes in Leucocyte Migration after Acute Hepatitis.Follow-up studies were performed in two patients with uncomplicated acute hepatitis by using autologous serum and liver obtained at the time of hepatitis as antigen (Fig. 4). During the acute phase of the illness cell-mediated immunity could be found against both antigens. This became weaker during convalescence and could not be detected by this technique three months after the start of the illness.

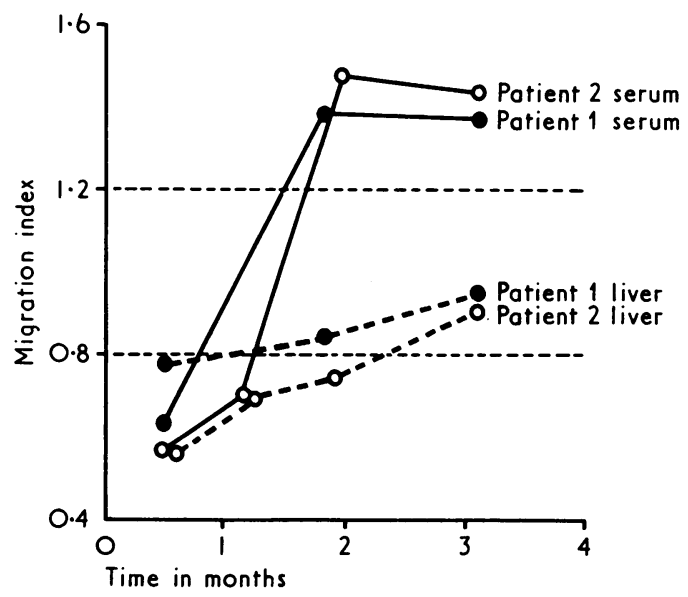

FIG. 4-Changes in migration index of leucocytes from two patients with H.A.A.-positive acute hepatitis during convalescence. Both autologous H.A.A.-positive liver and serum were used as antigen. Normal range between broken rules. 
LEUCOCYTE MIGRATION OF H.A.A.-NEGATIVE PATIENT

Twenty-one H.A.A.-negative patients were studied. They were divided into two groups depending on the presence or absence of serum antibody to H.A.A. detected by the haemagglutination assay. When liver homogenate obtained from H.A.A.-positive patients was used as antigen no cell-mediated immunity could be found against this antigen in the patients with no detectable antibody to H.A.A. Of the eight patients with antibody to H.A.A., two showed definite evidence of cell-mediated immunity against the antigen (Fig. 5). Similar results were obtained when

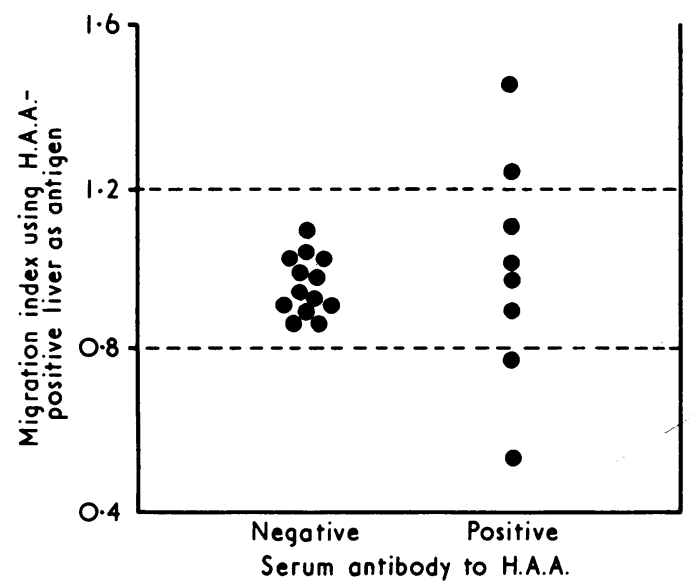
FIG. 5-Migration index of leucocytes from H.A.A.-negative patients either
with or without serum antibody to H.A.A. when H.A.A.-positive liver used with or without serum antibody to H.A.A. when
as antigen. Normal range between broken rules.

H.A.A.-positive serum was used as antigen. Stimulation of leucocyte migration was also noted occasionally but only in patients with antibody to H.A.A.

\section{Discussion}

The leucocyte migration test of Söborg and Bendixen has been used in man as an in-vitro assay to detect the presence of cellmediated immunity to a variety of test antigens (Bendixen and Söborg, 1969; Becker et al., 1972; Smith et al., 1972). Inhibition of leucocyte migration in the presence of antigen has been shown to be a good index of the presence of cell-mediated immunity to that antigen (Söborg, 1967). Stimulation of migration has also been described in this test system and it has been suggested that this represents weak cell-mediated immunity to the test antigen (Söborg, 1968). This conclusion, however, is still to be confirmed. In this study we have regarded only inhibition of leucocyte migration as definite evidence for the presence of cell-mediated immunity to the test antigen.

By using this test system we have been able to show cellmediated immunity in a number of patients to antigens contained in both the serum and liver of H.A.A.-positive patients. This cell-mediated immunity was found by using both autologous and homologous H.A.A.-positive test antigens, but was not found when the antigens were prepared irom H.A.A.-negative patients. Thus it appears likely that the cell-mediated immunity found in this study is specific for H.A.A. or other antigens related to the infective agent responsible for long incubation (type B) hepatitis. This is supported by the finding that cellmediated immunity to the H.A.A.-positive test antigens could be detected only in patients who had evidence of previous contact with H.A.A. as shown by the presence of H.A.A. or antibody to H.A.A. in their serum.

Cell-mediated immunity to the H.A.A.-positive test antigens was not detected in all the present H.A.A.-positive patients. It was found in all but one of the patients with acute hepatitis who subsequently recovered and cleared H.A.A. from their serum but was detected less frequently in patients with persistent H.A.A. in their serum. Cell-mediated immunity to these antigens was found in about half of the patients with chronic aggressive hepatitis or cirrhosis, was rare in those with chronic persistent hepatitis, and was never found in the apparently healthy carrier of H.A.A. Dudley et al. (1972) has postulated that a normal cellular immune response to antigenic determinants of the infective agent responsible for H.A.A.-positive hepatitis is necessary for the production of an acute hepatitis with subsequent recovery and clearance of antigen. However, in the presence of an impaired cellular immune response to these antigens both the infective agent and H.A.A. will persist and the activity of the associated liver cell damage will be less in the patients with the greater impairment of this response. Our results support this hypothesis.

Cell-mediated immunity to the H.A.A.-positive test antigen could be found in only some of the patients with serum antibody to H.A.A., and disappeared during the convalescence of two patients after wute hepatitis. This could mean that cellmediated immunity to our test antigens does not persist after hepatitis, or, more likely, that the test as used in this study is of insufficient sensitivity to detect weak cell-mediated immunity.

Other reports of leucocyte migration to varied antigens in liver disease tend to support our results. When H.A.A. concentrated from sera by ultracentrifugation was used as test antigen (Ito et al., 1972) cell-mediated immunity was commonly detected in patients with a history of H.A.A.-positive acute hepatitis but could not be detected in five patients with persistent antigenaemia (four with chronic hepatitis). This again suggests the importance of cell-mediated immunity in the clearance of the infective agent responsible for H.A.A.-positive hepatitis. In another study liver antigen prepared from normal fetal or postmortem liver was used as test antigen (Smith et al., 1972) and cell-mediated immunity was definitely found to this antigen in only one of 15 patients with acute hepatitis. This study supports our findings that the cell-mediated immunity found in the present patients is specific for antigens related to the infective agent associated with H.A.A.

This study has thus shown the presence of cell-mediated immunity to antigens related to the infective agent responsible for H.A.A.-positive hepatitis in a number of patients with evidence of earlier contact with this agent. Dudley et al. (1972) postulated that the liver cell damage in H.A.A.-positive patients is related to the $T$ lymphocyte cell-mediated immune response against the associated infective agent and that variations in $T$ lymphocyte function may be responsible for the different clinical courses that can follow contact with this infective agent. Our results support this hypothesis.

F.J.D. was supported by a grant from the Wellcome Foundation.

Requests for reprints should be addressed to: Professor S. Sherlock, Department of Medicine, Royal Free Hospital, London WC1X 8LF.

\section{References}

Becker, F. W., Krull, P., Deicher, H., and Kalden, J. R., (1972). Lancet 1, 120.

Bendixen, G., and Söborg, M. (1969). Danish Medical Bulletin, 16, 1.

Dudley, F. J., Fox, R. A., and Sherlock, S. (1972). Lancet, 1, 723.

Fox, R. A., Niazi, S. P., and Sherlock, S. (1969). Lancet, 2, 609.

Giles, J. P., McCollum, R. W., Berndtson, L. W. jun., and Krugman, S. (1969). New England fournal of Medicine, 281, 119 .

Gocke, D. I. and Howe, C. (1970). Fournal of Immunology, 104, 1031.

Ito, K., Nakagawa, J., Okimoto, Y., and Nakano, H. (1972). New England Tournal of Medicing, Okimoto,

Krugman, S., and Giles, J. P. (1970). fournal of the American Medical Association, 212, 1019 .
rugman,

Söborg, M. (1967). Acta Medica Scandinavica, 182, 167.

Söborg, M. (1968). Acta Medica Scandinavica, 184, 135.

Söborg, M. (1971). In-vitro Methods in Cell-mediated Immunity, ed. B. R. Bloom and P. R. Glade, p. 289. London, Academic Press.

Söborg, M., and Bendixen, G. (1967). Acta Medica Scandinavica, 181, 247. Smith, M. G. M., et al. (1972). British Medical fournal, 1, 527. 